\title{
Revisión
}

\section{REVISIÓN CRÍTICA DE LA LITERATURA SOBRE LAS NECESIDADES NO CUBIERTAS DE PERSONAS SUPERVIVIENTES DE CÁNCER}

\section{CRITICAL REVIEW OF THE LITERATURE ON THE UNMET NEEDS OF CANCER SURVIVORS}

Irati Padura Blanco. Hospital Galdakao-Usansolo. Osakidetza-Servicio Vasco de Salud. Galdakao, Vizcaya.

Ainhoa Ulibarri Ochoa. Escuela Universitaria de Enfermería de Vitoria-Gasteiz. Osakidetza-Servicio Vasco de Salud

Autor de correspondencia: ainhoa ulibarri@ehu.eus

\section{RESUMEN}

Introducción: La población de personas supervivientes de cáncer es cada vez mayor a nivel mundial. En España se estima que existen alrededor de 1.500 .000 supervivientes de cáncer. Muchas personas perciben este momento de fin de los tratamientos con miedo y con sentimientos de abandono y desprotección. Algunos estudios ponen de manifiesto los efectos secundarios a largo plazo y las alteraciones psicológicas y desvelan que con frecuencia las necesidades de estas personas no se encuentran bien cubiertas.

Objetivo: Identificar y describir las necesidades no cubiertas de personas supervivientes de cáncer en la fase extendida y permanente.

Método: Revisión crítica de la literatura en las bases de datos Medline, CINAHL, PsycINFO,
Cochrane, Embase y Cuiden y búsqueda manual en las revistas Journal of Cancer Survivorship y European Journal of Cancer Care. Criterios de inclusión: estudios publicados en los últimos 10 años (2009-2019); en castellano e inglés; adultos > 18 años en la fase de supervivencia extendida o permanente. Para asegurar la calidad de los estudios se utilizaron los criterios de las guías CASPe. Se realizó un análisis por categorías agrupando la información relevante.

Resultados: De los 1.035 artículos encontrados en la búsqueda, un total de 17 artículos fueron incluidos en el análisis final. Los hallazgos evidencian tres tipos de necesidades no cubiertas: físicas, psicosociales y espirituales. Estas personas necesitan recibir soporte para hacer frente a diferentes secuelas físicas como el cansancio, dolor, alteraciones digestivas, chemobrain, cambios 
en la imagen física y en la esfera sexual. A nivel psicosocial necesitan superar el cambio en el autoconcepto $y$ en los roles habituales, el miedo a la recidiva, el malestar emocional y a veces el aislamiento social. Los estudios también recogen necesidades espirituales no cubiertas como la necesidad de redefinir el significado de la vida y replantearse las prioridades.

Conclusiones: Las personas supervivientes de cáncer se enfrentan a una "nueva normalidad" a la que tienen que adaptarse con frecuencia sin la ayuda de los profesionales, ya que a partir del fin de los tratamientos el seguimiento es más espaciado y con frecuencia solo dirigido a la realización de pruebas para la detección precoz de posibles recidivas. Los resultados evidencian que las personas supervivientes necesitan una atención integral y continuada que responda a sus necesidades. Es necesario crear espacios donde poder llevar a cabo una valoración integral de estas necesidades y diseñar intervenciones para dar respuesta de manera multidisciplinar.

Palabras clave: Supervivientes de cáncer; necesidades no cubiertas; cáncer.

\section{ABSTRACT}

Introduction: The population of cancer survivors is increasing worldwide. In Spain, it is estimated that there are around $1,500,000$ cancer survivors. Many people perceive this moment of ending the treatments with fear and feelings of abandonment and lack of protection. Some studies highlight long-term side effects and psychological disturbances and reveal that the needs of these people are often not well met.

Aim: To identify and describe the unmet needs of cancer survivors in the extended and permanent phase.

Method: Critical review of the literature in the Medline, CINAHL, PsycINFO, Cochrane, Embase and Cuiden databases and manual search in the Journal of Cancer Survivorship and European Journal of Cancer Care. Inclusion criteria: studies published in the last 10 years (2009-2019); in Spanish and English; adults > 18 years in the extended or permanent survival phase. To ensure the quality of the studies, the criteria of the CASPe guidelines were used. A category analysis was performed, grouping the relevant information.

Results: Of the 1,035 articles found in the search, a total of 17 articles were included in the final analysis. The findings reveal three types of unmet needs: physical, psychosocial and spiritual. These people need to receive support to face different physical consequences such as tiredness, pain, digestive disturbances, chemobrain, changes in the image and in the sexual sphere. At the psychosocial level, they need to overcome the change in selfconcept and habitual roles, fear of relapse, emotional distress and sometimes social isolation. The studies also include unmet spiritual needs such as the need to redefine the meaning of life and rethink the priorities.

Conclusions: Cancer survivors face a "new normal" to which they 
have to adapt frequently without the help of professionals, since after the end of the treatments the follow-up is more spaced and often only directed towards the realization of tests for the early detection of possible recurrences. The results show that survivors need comprehensive and continuous care that responds to their needs. It is necessary to create spaces where to carry out a comprehensive assessment of these needs and design interventions to respond in a multidisciplinary way.

Keywords: Cancer survivors; unmet needs; cancer.

\section{INTRODUCCIÓN}

El cáncer sigue constituyendo una de las principales causas de morbimortalidad, con aproximadamente 18,1 millones de casos nuevos y 9,5 millones de muertes a nivel mundial ${ }^{1}$. También en España el cáncer es una de las principales causas de morbi-mortalidad, estimándose 277.394 casos nuevos en el año $2020^{2}$.

El pronóstico de esta enfermedad depende de múltiples factores como la localización, el estadio, la diseminación orgánica y las características inmunofenotípicas y puede ser muy variable ${ }^{3}$. En los últimos años, no obstante, gracias a los avances en el diagnóstico y tratamiento, la tasa de mortalidad ha ido descendiendo progresivamente, aumentando, por ende, el número de supervivientes. A nivel mundial hoy día ya son 43,8 millones de personas supervivientes de cáncer a los cinco años desde el diagnóstico ${ }^{1}$. En España también la supervivencia se ha duplicado en los últimos 40 años, siendo probable que continúe aumentando lentamente en los próximos años ${ }^{2}$. En concreto, se estiman alrededor de 1.500 .000 supervivientes en España ${ }^{4}$, con una supervivencia neta a los 5 años de los pacientes diagnosticados en el período 20082013 de $55,3 \%$ en los hombres y $61,7 \%$ en las mujeres ${ }^{2}$.

En el ámbito oncológico, el concepto de superviviente fue definido por primera en vez en 1985 por Fitzhugh Mullan, médico y paciente oncológico, quien estableció tres fases diferentes en la supervivencia: la supervivencia aguda (acute survival) que engloba las diferentes pruebas diagnósticas y el comienzo del tratamiento; la supervivencia extendida (extended survival) que comprende el período en el que la enfermedad comienza a remitir y se finaliza el tratamiento primario; y por último, la supervivencia permanente (permanent survival) establecida como la cura o remisión de dicha enfermedad ${ }^{5}$.

A lo largo de la vivencia de esta enfermedad son muchos los momentos de gran impacto para la persona, experimentando miedo al diagnóstico y al pronóstico, miedo a los efectos secundarios derivados de los diferentes tratamientos, inseguridad con respecto a los cambios funcionales y cambios en el desempeño de los roles habituales, entre otros. Si bien se pudiera pensar que el fin de la fase activa del tratamiento puede conllevar una disminución de la preocupación y la angustia, la realidad es bien diferente. Tal y como se recoge en el estudio canadiense "Living with cancer"6 publicado en 2018, los 
supervivientes se enfrentan a una "nueva normalidad" a la que tienen que adaptarse $y$ se preguntan cómo será la vida después, si dispondrán de la ayuda necesaria, si tendrán una recidiva. Después de finalizar el tratamiento, las personas sufren varios cambios, algunos de los cuales persisten varios años y otros se vuelven una parte permanente en su vida, por lo que estas personas y sus allegados tienen que afrontar una realidad en ocasiones muy diferente a su vida antes del cáncer y muchas personas perciben este momento de fin de los tratamientos con miedo y con sentimientos de abandono y desprotección.

El Grupo Español de Pacientes con Cáncer (GEPAC) ${ }^{7}$ en su "Informe sobre las necesidades de los supervivientes de cáncer" realizado en 2012 , documenta que las personas supervivientes de cáncer experimentan efectos secundarios a largo plazo en las fases de supervivencia extendida y permanente, acompañados de alteraciones psicológicas, y con frecuencia, tienen necesidades que no se encuentran todavía bien cubiertas.

A pesar de que cada vez son más las iniciativas y planes dirigidos a los supervivientes de cáncer ${ }^{8-10}$, en la práctica clínica diaria la atención al superviviente de cáncer todavía no está consensuada y existe un gran vacío en el seguimiento de este grupo de personas una vez finalizada la fase de tratamiento activo $^{11}$. Esto se debe a que la atención se centra en la supervivencia, más que en las vidas de aquellos que han sobrevivido al cáncer ${ }^{12}$. Por tanto, previo a diseñar planes de seguimiento e intervención que aborden a la persona de forma integral, es necesario conocer en mayor profundidad cuáles son las necesidades únicas y no cubiertas de las personas supervivientes de cáncer.

\section{OBJETIVO}

El objetivo de la presente revisión es identificar y describir las necesidades no cubiertas de personas supervivientes de cáncer en la fase extendida (después del fin de los tratamientos) o permanente (supervivencia prolongada).

\section{MÉTODO}

\section{Diseño}

Para dar respuesta al objetivo se realizó una revisión crítica de la literatura.

\section{Estrategia de búsqueda}

En primer lugar, se desglosó el objetivo en conceptos básicos (cáncer, supervivientes y necesidades no cubiertas) y se identificaron sinónimos, traduciendo todo al inglés mediante la aplicación DeCS. Cada concepto se convirtió a lenguaje controlado mediante los tesauros de las diferentes bases de datos.

A continuación, se combinaron los diferentes conceptos y se diseñaron las ecuaciones de búsqueda, aplicando diversos límites de acuerdo a los criterios de inclusión y exclusión para afinar la búsqueda y responder al objetivo de la revisión.

La búsqueda se llevó a cabo en las bases de datos Medline, CINAHL, PsycINFO, Cochrane, Embase y Cuiden. También se realizó búsqueda manual en las revistas 
Journal of Cancer Survivorship y European Journal of Cancer Care, así como en las guías de buenas prácticas de la Registered Nurses' Association of Ontario (RNAO) y en las guías de práctica clínica del National Institute for Health and Care Excellence (NICE). El proceso de búsqueda se desarrolló desde octubre de 2019 hasta febrero de 2020.

\section{Criterios de inclusión y exclusión}

Criterios de inclusión:

- Tipo de publicación: artículos originales (cuantitativos $y$ cualitativos), revisiones sistemáticas, metaanálisis, revisiones de la literatura y guías de práctica clínica.

- Fecha de publicación: últimos 10 años (20092019).

- Idioma: castellano e inglés.

- Población a estudio: Adultos mayores > 18 años en la fase de supervivencia extendida o permanente.

Criterios de exclusión:

- Tipo de publicación: artículos de opinión, editoriales, tesis, trabajos fin de grado o cartas al director.

- Fecha de publicación: publicaciones previas al 2009.

- Población a estudio: pacientes oncológicos pediátricos y adultos $>18$ años que se encuentren en la fase de supervivencia aguda.

- No disponer del artículo a texto completo.

\section{Proceso de búsqueda y selección de los artículos}

En una primera fase, en la búsqueda inicial se obtuvieron 1.035 artículos aplicando los criterios de inclusión de fecha, tipo de publicación y población a estudio, pero 195 estaban duplicados, con lo que inicialmente se partió de 840 artículos. En la lectura de los títulos y abstract se excluyeron 534 artículos por no responder al objetivo y no cumplir con los criterios de idioma y disponibilidad del texto completo.

En una segunda fase, de los 306 artículos a texto completo se excluyeron 290 por no responder al objetivo o no cumplir con los criterios de inclusión de tipo de publicación y población a estudio, seleccionando 16 artículos. A estos se añadió un artículo de la búsqueda manual obtenido de la revista Journal of Cancer Survivorship.

Finalmente, en una tercera fase, los 17 artículos fueron sometidos a análisis crítico mediante un guion de lectura crítica basado en los criterios de las guías CASPe para asegurar su calidad. Los 17 artículos fueron seleccionados: 11 cuantitativos descriptivos (10 transversales y uno longitudinal), 3 cualitativos, 2 con diseño mixto y un metaanálisis. Ver diagrama de flujo PRISMA del proceso de búsqueda y selección de la literatura en la Figura 1. 


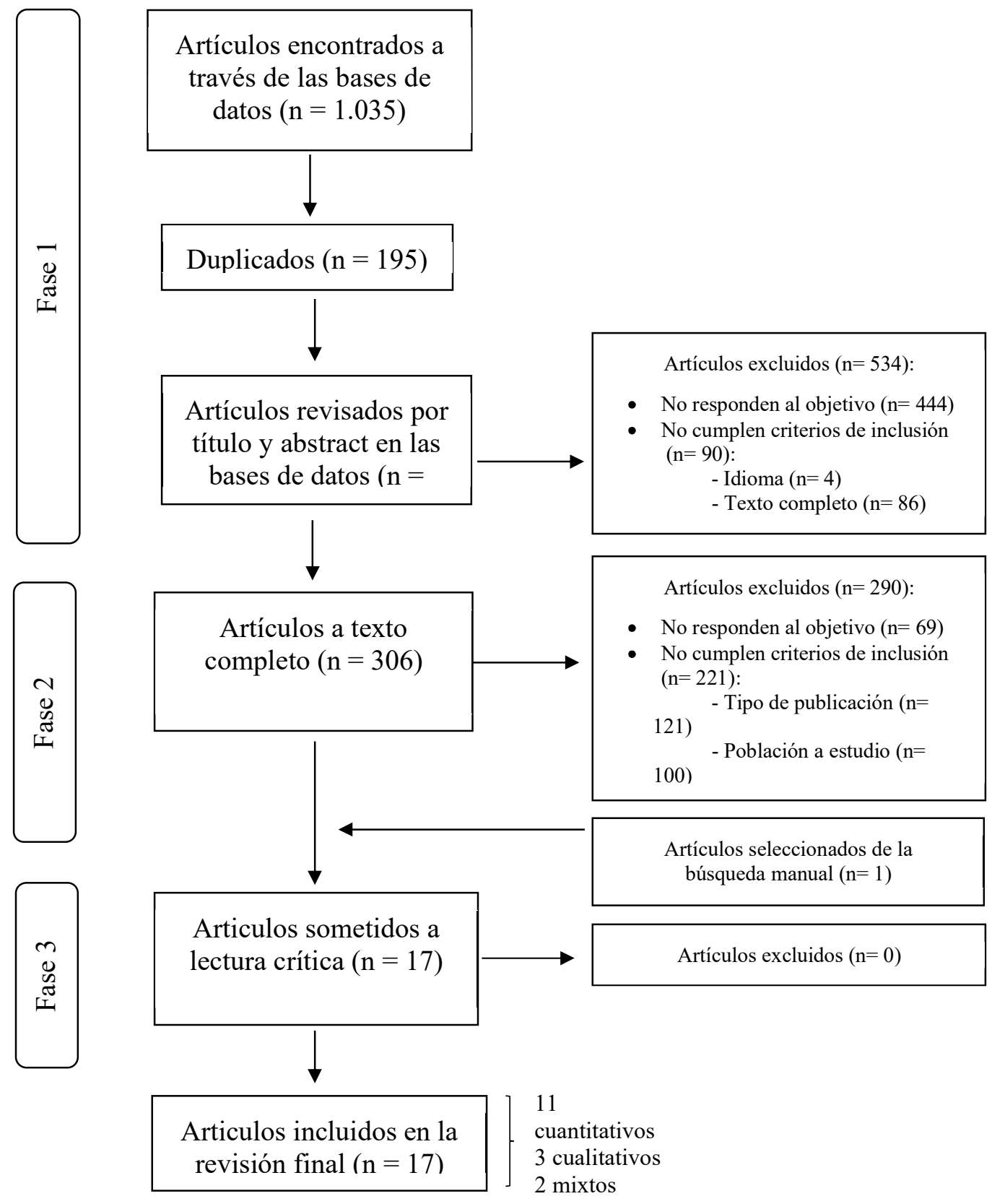

Medline: 6; Cinahl: 4; PsycINFO: 2; Embase: 4

+ Journal of Cancer Survivorship: 1

Figura 1. Diagrama de flujo PRISMA del proceso de búsqueda y selección de la literatura. 


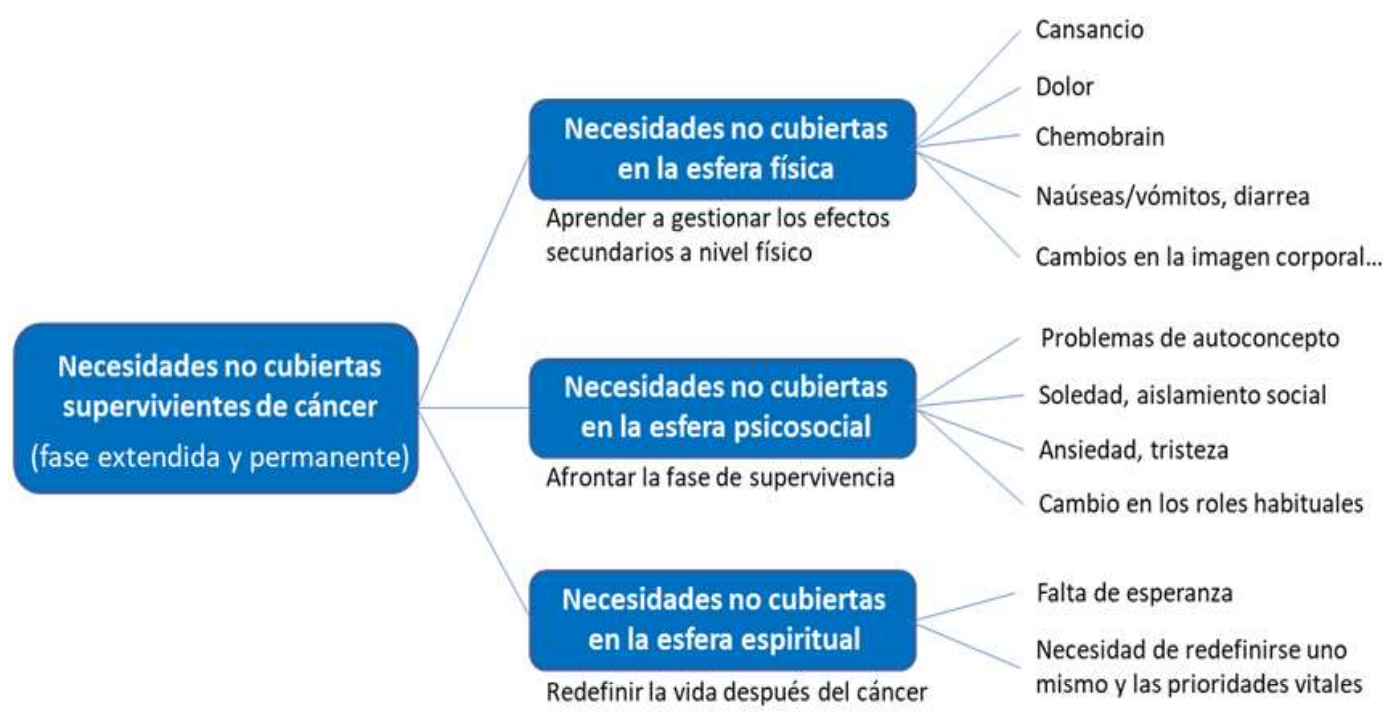

Figura 2. Árbol categorial.

\section{Análisis e interpretación de la información}

A partir de los 17 artículos seleccionados se realizó un preanálisis del contenido de los mismos y seguidamente se realizó una lectura detallada agrupando en una tabla resumen la información relevante para responder al objetivo de la presente revisión (Tabla ANEXO 1).

Finalmente, la información relevante se organizó en diferentes categorías elaborando un árbol categorial (Figura 2) y se procedió a realizar la interpretación de los resultados.

\section{RESULTADOS}

A partir de la revisión de la literatura se seleccionaron
17 artículos, gran parte de ellos (65\%) artículos cuantitativos descriptivos (Figura 3). Todos ellos fueron publicados entre los años 2013 y 2019 (Figura 4) en diferentes países, con una mayor publicación en EEUU y China (Figura 5).

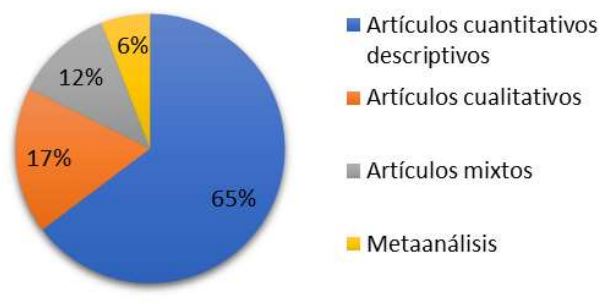

Figura 3. Diseño de los artículos. Fuente: Elaboración propia. 


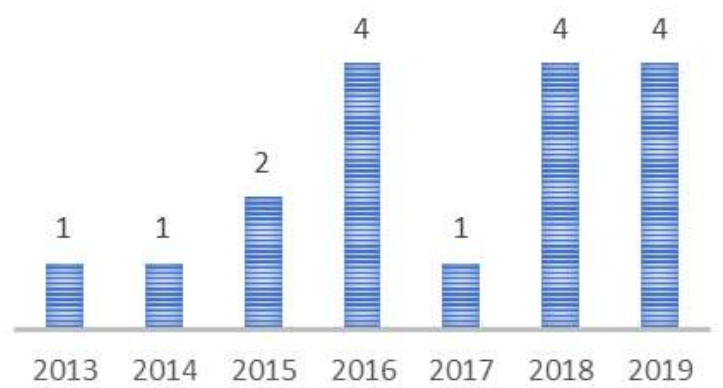

Figura 4. Artículos por año. Fuente: Elaboración propia. supervivientes de cáncer y la necesidad de que las personas aprendan a gestionar los efectos secundarios a largo plazo.

Una de las mayores dificultades descritas por los supervivientes es el cansancio y las limitaciones que éste impone en el desarrollo de la vida diaria. Tres estudios recientemente publicados así lo recogen. Pongthavornkamol et al. ${ }^{13}$ estudiaron la prevalencia de las afectaciones físicas en 236 pacientes de diversas localizaciones (mama, pulmón,

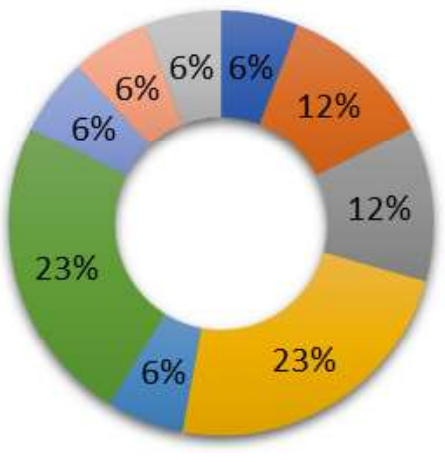

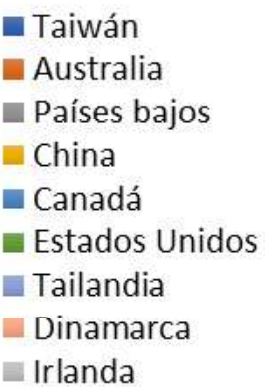

Figura 5. Artículos por países. Fuente: Elaboración propia.

En relación con el contenido, los artículos de esta revisión abordan 3 ámbitos de necesidades no cubiertas en los supervivientes de cáncer: necesidades no cubiertas en la esfera física, en la esfera psicosocial y en la esfera espiritual.

\section{Necesidades no cubiertas en la esfera física}

Son varios los autores que han descrito las diversas afectaciones físicas presentes en los testicular, colorrectal...) 1-5 años después de haber finalizado el tratamiento activo utilizando la escala validada "Cancer Survivor Survey of Needs". El cansancio fue una de las afectaciones más frecuentes en un $43,2 \%$ de los participantes, relacionándose con una baja calidad de vida en los pacientes de mama, colorrectal y pulmón. Otros dos estudios también han reportado el cansancio como uno de los efectos secundarios a largo plazo con los que las personas supervivientes tienen que aprender a convivir. En 
concreto, So et al. ${ }^{14}$ en un estudio con 53 supervivientes de cáncer de cabeza y cuello objetivaron que el cansancio, la falta de energía y el malestar general estaban presentes en el $32 \%$ de los participantes, dificultándoles el llevar a cabo las actividades de la vida diaria. Lo et al. ${ }^{15}$ también identificaron el cansancio, agotamiento físico y malestar en una muestra de 120 supervivientes, estando presente en el $85 \%$ de los participantes.

Con relación al cansancio, agotamiento y malestar, los supervivientes hacen un llamamiento al sistema sanitario para poder hacerles frente. Partiendo de la herramienta validada The Cancer Survivors' Unmet Need Measure" (CaSUN), se percibió que los supervivientes refieren la necesidad de recibir más información (oral, escrita, mediante diagramas o de manera audiovisual) para afrontar esta situación de manera autónoma en sus hogares, pero sin dejar a un lado el seguimiento y valoración continuada por parte de los profesionales de la salud ${ }^{13-15}$.

Otra de las afectaciones más frecuentes manifestadas por los supervivientes es el dolor. En el estudio de Manne et al. ${ }^{16}$ un $12 \%$ de los participantes refirió que el dolor estaba presente después de la fase aguda de la enfermedad, extendiéndose durante un largo período después de finalizado el tratamiento primario. Reb y Cope ${ }^{17}$ también identificaron el dolor como uno de los síntomas más recurrentes en los supervivientes, ocupando la décima posición en un total de 41 síntomas descritos por mujeres supervivientes de cáncer ginecológico. Los resultados de este estudio además confirmaron la relación entre la intensidad y duración del dolor con la localización tumoral. Así, los supervivientes de cáncer de pulmón referían mayor intensidad de dolor durante períodos más largos. En este sentido, Wang et al. ${ }^{18}$ objetivaron que un $46,2 \%$ de los pacientes describía que la atención recibida por parte de los sanitarios era insuficiente y que la analgesia recetada para gestionar el dolor era escasa.

Al cansancio físico y dolor se añade el fenómeno denominado Chemobrain, que consiste en el deterioro de la función cerebral con problemas de memoria a corto plazo y dificultades en la concentración a la hora de estudiar, trabajar o incluso a la hora de leer. Este fenómeno fue descrito por el $36 \%$ de los participantes en el estudio de Pongthavornkamol et al. ${ }^{13}$. Los supervivientes refirieron no recibir información ni consejos para poder sobrellevar esta condición. Según Den Bakker et al. ${ }^{19}$, esta falta de información y de asesoramiento deja al descubierto la necesidad de crear guías consensuadas que trabajen las afectaciones recurrentes, para dejar a un lado el vacío asistencial descrito por tantos supervivientes ("I miss the aftercare").

Los problemas digestivos tales como cuadros nauseosos acompañados de emesis y diarreas son otra de las grandes afectaciones que más interfieren en la calidad de vida de los supervivientes ${ }^{13,17}$. Sin embargo, se ha podido observar que dichas afectaciones gastrointestinales no afectan de igual manera a todas las personas. En el estudio 
realizado en Australia por Hardcastle et al. ${ }^{20}$ con supervivientes de cáncer colorrectal, se objetivó que los cambios en la motilidad suponían, por delante de otras afectaciones, la mayor preocupación. Sin embargo, en el estudio de Pongthavornkamol et al. ${ }^{13}$, donde las localizaciones del cáncer eran diversas, estos cambios fueron descritos únicamente por el 12,7\% de los participantes. Ante estas afecciones digestivas también el asesoramiento y acompañamiento recibidos han sido descritos como insuficientes. En concreto, en la publicación de Willems et al. ${ }^{21}$ el $25,9 \%$ afirmó necesitar ayuda para hacer frente a estos habituales efectos secundarios. De igual manera, expresaron la necesidad de apoyo sanitario para adoptar estilos de vida saludables como, por ejemplo, qué dieta o ejercicios pudieran ser beneficiosos para hacer frente a los cambios de peristaltismo ${ }^{20}$.

Para finalizar con las afectaciones físicas más frecuentes y las necesidades derivadas de las mismas, se ha podido observar que, aunque en ocasiones se pase por alto, los cambios que se sufren en la imagen corporal suponen alteraciones notorias entre los supervivientes. Lo et al. ${ }^{15}$ encontraron que el $77 \%$ de los supervivientes de su estudio describieron pérdida del pelo, cambios en el peso y cambios en la piel (pigmentación, sequedad), con un gran impacto en su bienestar. En el estudio de Manne et al. ${ }^{16}$ más de un $20 \%$ de los participantes mostraron gran preocupación ante esta realidad. Además, algunos autores han relacionado los cambios en la imagen corporal con dificultades en el ámbito sexual como la dispareunia, provocando incluso crisis en la pareja ${ }^{13,14}$. En este sentido, algunos estudios evidencian que al igual que pasa con la distorsión en la imagen corporal, las dificultades que pueden darse en el ámbito sexual en muchas ocasiones son pasadas por alto. Así, Wang et al. ${ }^{18}$ afirman que la información y el apoyo ante las dificultades del ámbito sexual son escasos o nulos. En este sentido, So et al. ${ }^{22}$ recogen un testimonio muy significativo de una mujer superviviente de cáncer de mama: "...We want someone to tell us wether it's OK to have sex. But no one will tell you that" ( ....Queremos que alguien nos diga que está bien tener sexo, pero nadie te dirá eso").

En los diferentes estudios se ha observado que los supervivientes solicitan continuamente recibir información acerca de los efectos secundarios que pueden presentar a corto y largo plazo una vez terminado el tratamiento activo, qué cuidados pueden llevar a cabo para manejarlos $o$ ante qué síntomas deberían mantenerse alerta y acudir al sistema sanitario 23 .

\section{Necesidades no cubiertas en la esfera psicosocial}

Ciertas afectaciones a nivel físico tienen una relación directa en la esfera psicosocial. Cambios en la imagen corporal, tales como la pérdida de pelo o alteraciones en el peso, pueden llegar a generar distorsiones en el autoconcepto creando sentimientos de vergüenza y menosprecio hacia uno mismo ${ }^{17,24}$. Esto unido a ciertas alteraciones en la esfera física como el agotamiento pueden hacer que los supervivientes se 
aíslen, dificultando en ocasiones las interacciones sociales ${ }^{25}$.

Los supervivientes de cáncer también refieren con frecuencia ansiedad y tristeza, así como miedo a posibles recidivas, miedo a la muerte y miedo a un futuro incierto. Simples resfriados y malestares que en situaciones "normales" podrían pasar inadvertidos, pueden crear altos niveles de ansiedad en los supervivientes por temer que pudieran ser indicios de recaídas ${ }^{16}$. En este sentido, Ellegaard et al. ${ }^{26}$ en su estudio con 155 mujeres supervivientes de cáncer de mama identificaron la carencia de programas de seguimiento para cubrir las necesidades relacionadas con las dudas posttratamiento $y$, por ende, los miedos durante la fase de supervivencia.

So et al. ${ }^{14}$ por su parte descubrieron que los cambios sufridos a nivel físico y su duración a lo largo del tiempo podían causar preocupación debido a la ruptura en los roles habituales. El traspaso de funciones o cambio de roles crea una sensación de "inutilidad" al mismo tiempo que soledad, a pesar de disponer del apoyo de familiares o amigos cercanos, ya que es difícil que éstos verdaderamente lleguen a comprender, por mucho que se esfuercen, la vivencia del que lo padece si no se ha vivido previamente en primera persona. El sentimiento de soledad también se puede sentir a la hora de "reintegrarse" en la sociedad, ya que todavía hoy predomina una connotación negativa de la enfermedad $^{25}$. Con motivo de hacer frente a todas estas cuestiones, algunos estudios recogen que los supervivientes perciben la necesidad de terapias grupales donde personas que hayan pasado por procesos oncológicos puedan compartir experiencias enriquecedoras o incluso recomendaciones que puedan ser una base de apoyo y acompañamiento para los participantes ${ }^{18,19}$

\section{Necesidades no cubiertas en la esfera espiritual}

Diferentes estudios también han identificado necesidades no cubiertas en la esfera espiritual. Algunos participantes han descrito sentirse perdidos, desesperanzados, sobre todo a partir de la fase de supervivencia extendida, en parte unido a que una vez terminada la fase de tratamiento comienzan a ver descender el número de citas con los profesionales que han permanecido a su lado durante todo el proceso previo de diagnóstico y tratamiento, comenzando a "tambalearse" la red terapéutica creada hasta entonces ${ }^{17}$.

Al mismo tiempo aparece la necesidad de "redefinirse" a uno mismo, replanteándose las prioridades vitales que hasta ese momento suponían un gran pilar ${ }^{17}$. Según Burg et al. ${ }^{24}$, esta necesidad de redefinirse y de replantearse los principios vitales radica en la connotación negativa que a día de hoy aún conserva esta enfermedad. La sociedad ve a estas personas como "enfermos" durante largos periodos pese a haber superado con creces la fase activa de la enfermedad. Es por eso por lo que en ocasiones aparecen dificultades a la hora de encontrarle "sentido" al proceso vivido $y$ de redefinirse ${ }^{24}$. En 
ocasiones, durante el proceso de redefinirse $y$ de replantearse tantas cosas, los supervivientes pueden mostrar flaquezas en sus creencias y valores.

En todo este proceso los supervivientes perciben la necesidad de tener al lado ayuda y apoyo mientras se exploran todos estos aspectos espirituales ${ }^{27}$.

Factores moduladores de las necesidades no cubiertas a nivel físico, psicosocial y espiritual

Varias publicaciones han mostrado que las necesidades no cubiertas en la esfera física, psicosocial y espiritual pueden ser moduladas por diversos factores:

- Edad: se ha identificado que las personas más jóvenes presentan más necesidades a nivel emocional ${ }^{15,24,26-28}$.

- Sexo: aunque existe controversia en si existen diferencias entre hombres y mujeres, Lo et al. ${ }^{15}$ encontraron mayor necesidad de información en los hombres frente a las mujeres. Por su parte, so et al. ${ }^{22}$ y Ellegaard et al. ${ }^{26}$ observaron que las mujeres se mostraban más independientes a la hora de buscar respuestas y soluciones a los problemas y que mantenían una mayor coordinación con los profesionales de la salud durante la fase de supervivencia.

- Nivel educativo: Cheng et al. $^{29}$ encontraron que, a mayor nivel educativo, mayores son las necesidades de las personas y las demandas al sistema sanitario. De las 250 mujeres supervivientes de cáncer de mama estudiadas, las $21,2 \%$ que albergaban títulos universitarios presentaron más necesidades en el ámbito psicológico y solicitaron más información sobre el periodo postterapéutico en comparación con aquellas con estudios primarios.

- Características de la enfermedad: se ha identificado que los supervivientes que han presentado recidivas muestran mayor necesidad de información $(p=0,001)$. Del mismo modo, aquellos con mayores afectaciones físicas como el dolor, el agotamiento o la pérdida de peso presentan mayor número de necesidades relacionadas con el cuidado $(p=<0,001)^{16}$.

- Fase de supervivencia: se ha identificado un posible nexo entre la fase de supervivencia en la que se encuentra el paciente y las necesidades que presenta. Cheng et al. ${ }^{29}$ observaron que, a mayor tiempo transcurrido desde el final del tratamiento, mayores son las necesidades relacionadas con el ámbito psicosocial. 


\section{CONCLUSIONES}

Los resultados de esta revisión han mostrado que en las personas supervivientes de cáncer las diferentes afectaciones prosiguen a pesar de haber finalizado el tratamiento activo, extendiéndose a las fases de supervivencia extendida y permanente. El final de la fase aguda supone una "nueva normalidad" a la que tienen que adaptarse con frecuencia sin la ayuda de los profesionales, ya que a partir del fin de los tratamientos el seguimiento es más espaciado y con frecuencia solo dirigido a la realización de pruebas para la detección precoz de posibles recidivas. Esta falta de seguimiento genera un sentimiento de soledad $y$ de abandono, y como consecuencia, se han identificado diferentes necesidades no cubiertas a nivel físico, psicosocial y espiritual.

Sin embargo, a nivel de la práctica clínica, no existen guías consensuadas basadas en la evidencia que faciliten el apoyo emocional y la información que solicitan los supervivientes de cáncer para poder responder a las diferentes necesidades que presentan a lo largo del tiempo ${ }^{25}$. Según la NCCN (National Comprehensive Cancer Network) ${ }^{30}$ los planes de seguimiento a largo plazo deben ser diseñados e implementados, basados en un equipo multidisciplinar, donde participen, entre otros, el equipo de enfermería, médicos, nutricionistas y trabajadores sociales. Es necesario proveer una atención integral y continuada que responda a sus necesidades, creando para ello espacios donde poder llevar a cabo una valoración integral de sus necesidades, para posteriormente diseñar intervenciones personalizadas.

Es importante además que los supervivientes dispongan de un profesional sanitario de referencia con el que poder contactar ante cualquier tipo de preocupación o miedo, al mismo tiempo que les facilite el acceso a los recursos sanitarios disponibles ${ }^{22}$. De acuerdo con la $\mathrm{NCCN}^{30}$, la Enfermería es una de las claves fundamentales en la atención sanitaria a los supervivientes de cáncer. En este sentido, la Oncology Nursing Society (ONS) ${ }^{31}$ ha descrito el rol de la enfermera oncológica para asegurar la calidad en el cuidado de estos supervivientes de cáncer. También se ha puesto de manifiesto la necesidad de desarrollar nuevos roles como es el caso de la enfermería de práctica avanzada especializada en oncología 32 .

Como limitaciones de esta revisión cabe mencionar la dificultad para acceder al texto completo de varios artículos que podían haber aportado mayor conocimiento. Es importante también señalar que la mayoría de la literatura revisada fue de corte transversal. Por tanto, las necesidades identificadas no pueden extrapolarse a periodos futuros, ya que únicamente representan las necesidades descritas en el momento concreto en el que se llevaron a cabo los diferentes estudios. Además, sería importante investigar en las necesidades no cubiertas de supervivientes de cáncer con muestras más homogéneas, considerando la especificidad de la localización tumoral y los tratamientos recibidos con sus efectos secundarios a largo plazo, ya que son factores que pueden 
interferir en la diferente percepción de las necesidades.

\section{REFERENCIAS:}

(1) WHO-World Health Organization. The Global Cancer Observatory. Cancer fact sheet. [Internet]. Lyon: IARC; 2019 [acceso 30 de diciembre de 2019]. Disponible en: https://gco.iarc.fr/today/data/factsheets/ populations/900-world-fact-sheets.pdf

(2) SEOM-Sociedad Española de Oncología Médica. Las cifras del cáncer en España 2020. [Internet]. Madrid: seom.org; 2020 [acceso 15 de febrero de 2020]. Disponible en: https://seom.org/seomcms/images/storie s/recursos/Cifras del cancer 2020.pdf

(3) Devita VT, Lawrence TS, Rosenberg SA. DeVita, Hellman, and Rosenberg's Cancer Principles and Practice of Oncology. $11^{\mathrm{a}}$ ed. Philadelphia: Wolters Kluwer; 2019.

(4) Brozos E, Vázquez F, Cueva J, Barón FJ. Supervivientes al cáncer en España: Situación actual. pp. 7-15. En: SEOMSociedad Española de Oncología Médica. Monográfico SEOM de Largos

Supervivientes en Cáncer. $1^{\text {a }}$ parte. [Internet]. Madrid: seom.org; 2012 [acceso 30 de diciembre de 2019]. Disponible en: http://www.seom.org/seomcms/images/s tories/recursos/infopublico/publicaciones/ MONOGRAFICO_SEOM_LARGOS_SUPERV IVIENTES_I.pdf

(5) Mullan F. Seasons of Survival: Reflections of a Physician with Cancer. N Engl J Med. 1985;313(4):270-273.

(6) Canadian partnership against cancer. Living with Cancer. A Report on the Patient experience [Internet]. Toronto: Canadian Partnership Against Cancer Corporation; 2018 [acceso 20 de enero de 2020]. Disponible: https://s22457.pcdn.co/wpcontent/uploads/2019/01/Living-withcancer-report-patient-experience-EN.pdf

(7) Barragan B, Jovell AJ. Informe sobre las necesidades de los supervivientes de cáncer. [Internet]. Madrid: GEPAC-Grupo Español de Pacientes con Cáncer; 2012 [acceso 30 de diciembre de 2019]. Disponible en: http://www.gepac.es/docs/informe_super vivientes.pdf

(8) Departamento de Salud del Gobierno Vasco. Plan oncológico de Euskadi 20182023 [Internet]. Vitoria-Gasteiz: GV;
2018 [acceso 13 de noviembre de 2019]. Disponible en: https://www.euskadi.eus/contenidos/info rmacion/plan_oncologico_2018_2023/es def/adjuntos/plan-oncologico-euskadi2018-2023.pdf

(9) Osakidetza-Servicio Vasco de Salud. Programa Paciente Activo [Internet]. 2018 [acceso 13 de enero de 2020] Disponible en: https://www.osakidetza.euskadi.eus/quees-el-programa-paciente-activo/ab84oescuida/es/

(10) SEOM-Sociedad Española de Enfermería Oncológica. Plan Integral de Atención a los Largos Supervivientes de Cáncer [Internet]. Madrid: seom.org; 2013 [acceso 13 de noviembre de 2019]. Disponible en: https://seom.org/seomcms/images/storie s/recursos/PLAN_INTEGRAL_LARGO_SUP ERVIVIEN TE.pdf

(11) Institute of Medicine and National Research Council. Defining cancer survivors and survivorship: From cancer patient to cancer survivor, lost in transition. Washington DC: National Academies Press; 2006.

(12) O'Connor M, O'Donovan B, Drummond F, Donnelly C. National Cancer Survivorship Needs Assessment. The unmet needs of cancer survivors in Ireland: A scoping review 2019. [Internet]. Cork: National Cancer Registry Ireland; 2019 [acceso 20 de enero de 2020]. Disponible en: https://www.ncri.ie/publications/research -reports/unmet-needs-cancer-survivorsireland-scoping-review-2019.

(13) Pongthavornkamol K, Lekdamrongkul P, Pinsuntorn P, Molassiotis A. Physical Symptoms, Unmet Needs, and Quality of Life in Thai Cancer Survivors after the Completion of Primary Treatment. Asia Pac J Oncol Nurs. 2019;6(4):363-371.

(14) So WKW, Wong CL, Choi KC, Chan CWH, Chan JCY, Law BMH, et al. A MixedMethods Study of Unmet Supportive Care Needs among Head and Neck Cancer Survivors. Cancer Nurs. 2019;42(1):6778.

(15) Lo WT, Yates P, Chan RJ. Unmet supportive care needs and symptom burden in Taiwanese cancer survivors who have completed primary treatment. Eur J Oncol Nurs. 2018:35:79-84.

(16) Manne S, Hudson SV, Baredes S, Stroup A, Virtue SM, Paddock L, et al. Survivorship care experiences, information, and support needs of patients with oral and oropharyngeal 
cancer. Head Neck. 2016; 38(1):E193546.

(17) Reb AM, Cope DG. Quality of Life and Supportive Care Needs of Gynecologic Cancer Survivors. West J Nurs Res. 2019;41(10):1385-1406.

(18) Wang JW, Shen Q, Ding N, Zhang TR, Yang ZQ, Liu C, et al. A qualitative exploration of the unmet psychosocial rehabilitation needs of cancer survivors in China. Psychooncology. 2016;25(8):9051012.

(19) Den Bakker CM, Schaafsma FG, Huirne JAF, Consten ECJ, Stockmann HBAC, Rodenburg $\mathrm{CJ}$, et al. Cancer survivors' needs during various treatment phases after multimodal treatment for colon cancer - Is there a role for eHealth? BMC Cancer. 2018; 18:1207.

(20) Hardcastle SJ, Maxwell-Smith C, Hagger MS, O'Connor M, Platell C. Exploration of information and support needs in relation to health concerns, diet and physical activity in colorectal cancer survivors. Eur J Cancer Care. 2018;27(1): e12679.

(21) Willems RA, Bolman CAW, Mesters I, Kanera IM, Beaulen AAJM, Lechner L. Cancer survivors in the first year after treatment: the prevalence and correlates of unmet needs in different domains. Psychooncology. 2016;25(1):51-57.

(22) So WKW, Chow KM, Chan HYL, Choi KC, Wan RWM, Mak SSS, et al. Quality of life and most prevalent unmet needs of Chinese breast cancer survivors at one year after cancer treatment. Eur J Oncol Nurs. 2014;18(3):323-328.

(23) Santin O, Murray L, Prue G, Gavin A, Gormley G, Donnelly M. Self-reported psychosocial needs and health-related quality of life of colorectal cancer survivors. Eur J Oncol Nurs. 2015;19(4):336-342.

(24) Burg MA, Adorno G, Lopez EDS, Loerzel $\mathrm{V}$, Stein K, Wallace $\mathrm{C}$, et al. Current unmet needs of cancer survivors: Analysis of open-ended responses to the American Cancer Society study of cancer survivors II. Cancer. 2015;121(4):623630.

(25) Raque-Bogdan TL, Lamphere B, Kostiuk M, Gissen M, Beranek M. Unpacking the layers: a meta-ethnography of cancer survivors' loneliness. J Cancer Surviv. 2019;13(1):21-33.

(26) Ellegaard MBB, Grau C, Zachariae R, Jensen $A B$. Fear of cancer recurrence and unmet needs among breast cancer survivors in the first five years. A crosssectional study. Acta Oncol. 2017;56(2):314-320.
(27) Giuliani M, McQuestion M, Jones J, Papadakos J, Le L. W., Alkazaz N, et al. Prevalence and nature of survivorship needs in patients with head and neck cancer. Head Neck. 2016;38(7):10971103.

(28) Smith $A B$, King $M$, Butow $P$, Luckett $T$, Grimison $\mathrm{P}$, Toner GC, et al. The prevalence and correlates of sup ortive care needs in testicular cancer survivors: A cross-sectional study. Psychooncology. 2013;22(11):2557-2564.

(29) Cheng KKF, Cheng HL, Wong WH, Koh C. A mixed-methods study to explore the supportive care needs of breast cancer survivors. Psychooncology. 2018;27(1):265- 271.

(30) Sanft T, Denlinger CS, Armenian S, Baker KS, Broderick G, DemarkWahnefried W, et al. Survivorship, Version 2.2019. Featured updates to the NCCN guidelines. J Natl Compr Cancer Netw. 2019;17(7):785-794.

(31) Ferrell BR, Virani R, Smith S, Juarez G. The role of Oncology Nursing to Ensure Quality Care for Cancer Survivors: A report Commissioned by the National Cancer Policy Board and Institute of Medicine. Oncol Nurs Forum. 2003;30(1):E1-E11.

(32) Corcoran S, Dunne M, McCabe MS. The role of Advanced Practice Nurses in cancer survivorship care. Semin Oncol Nurs. 2015;31(4):338-347. 

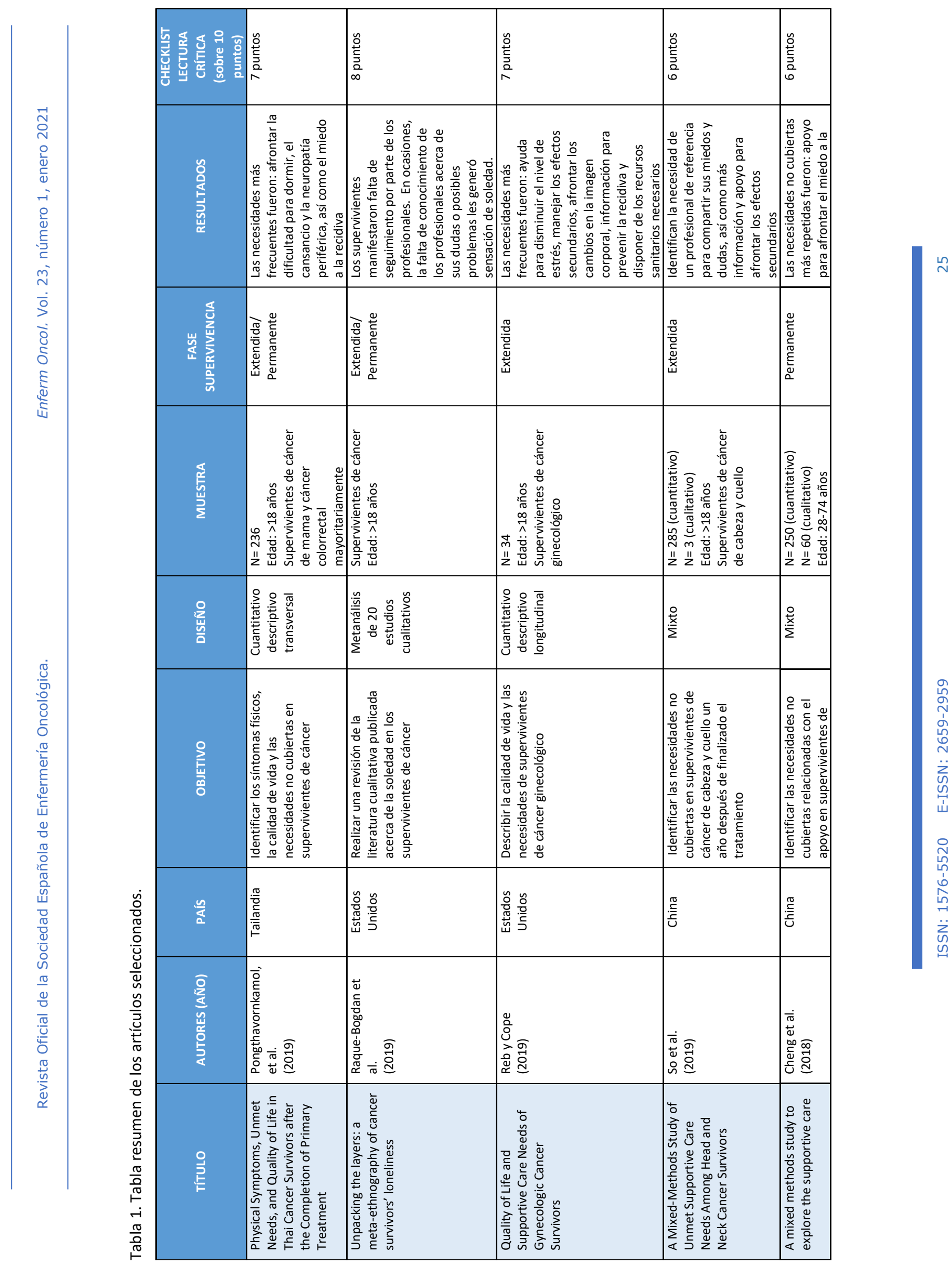


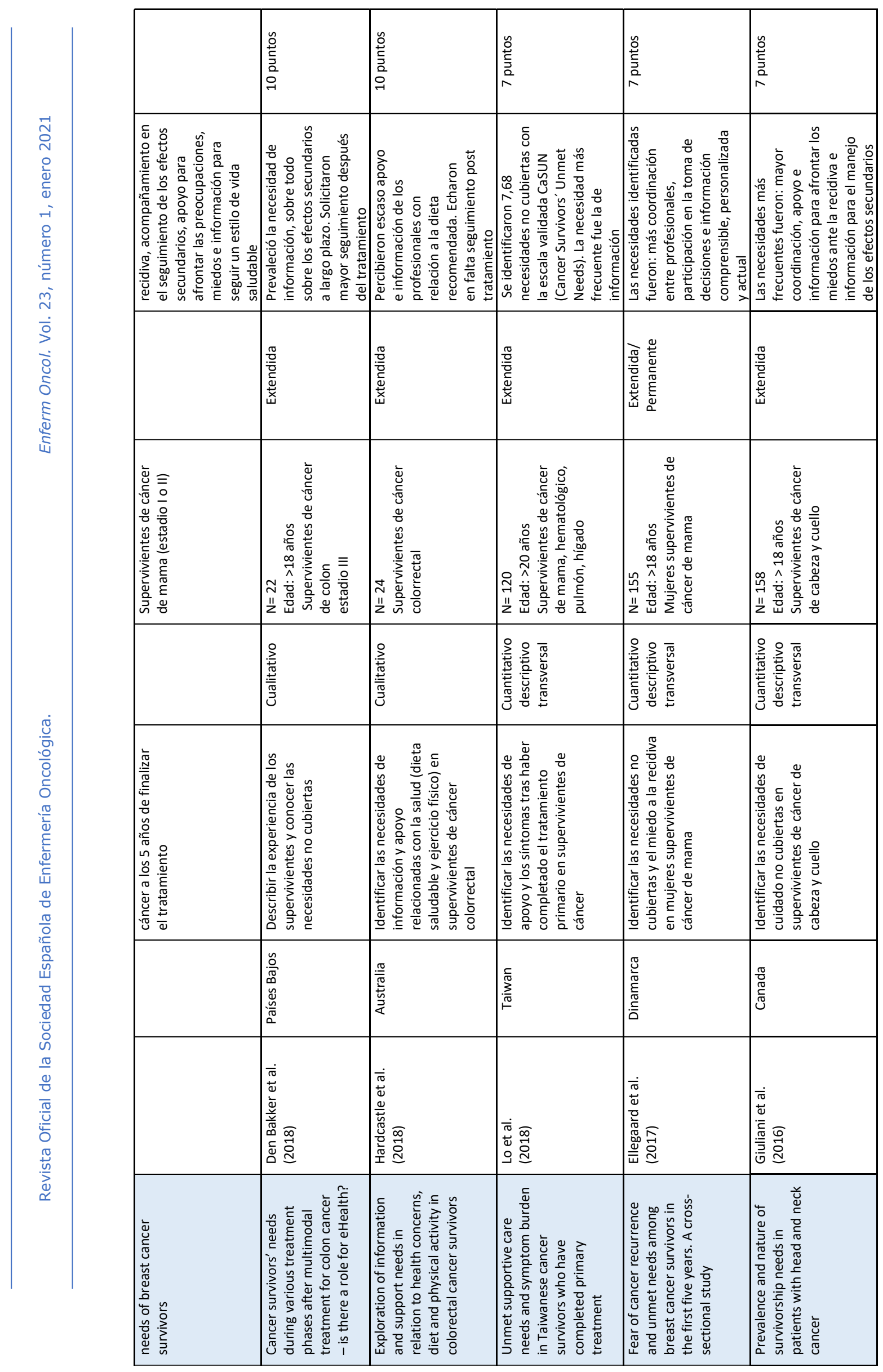




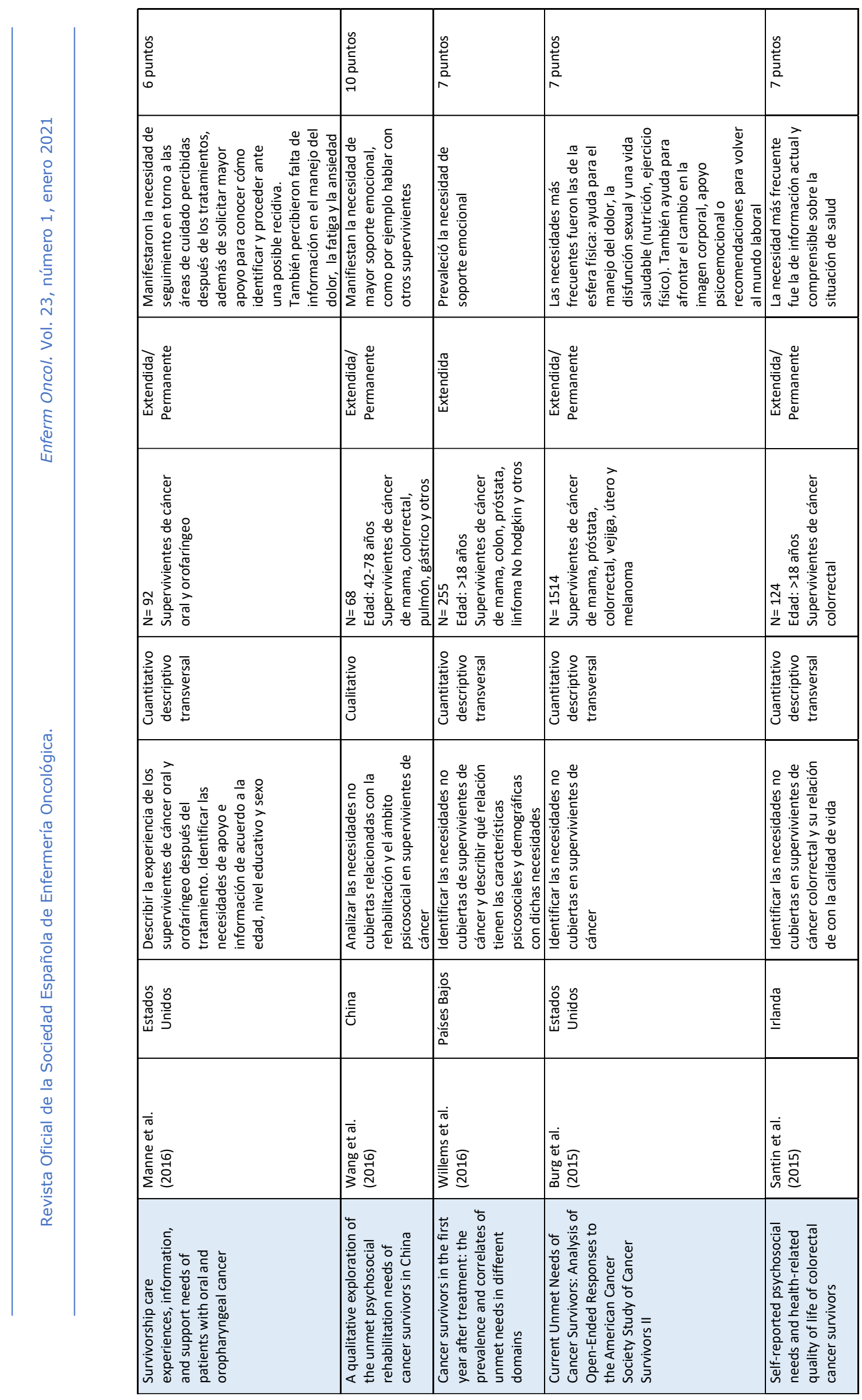




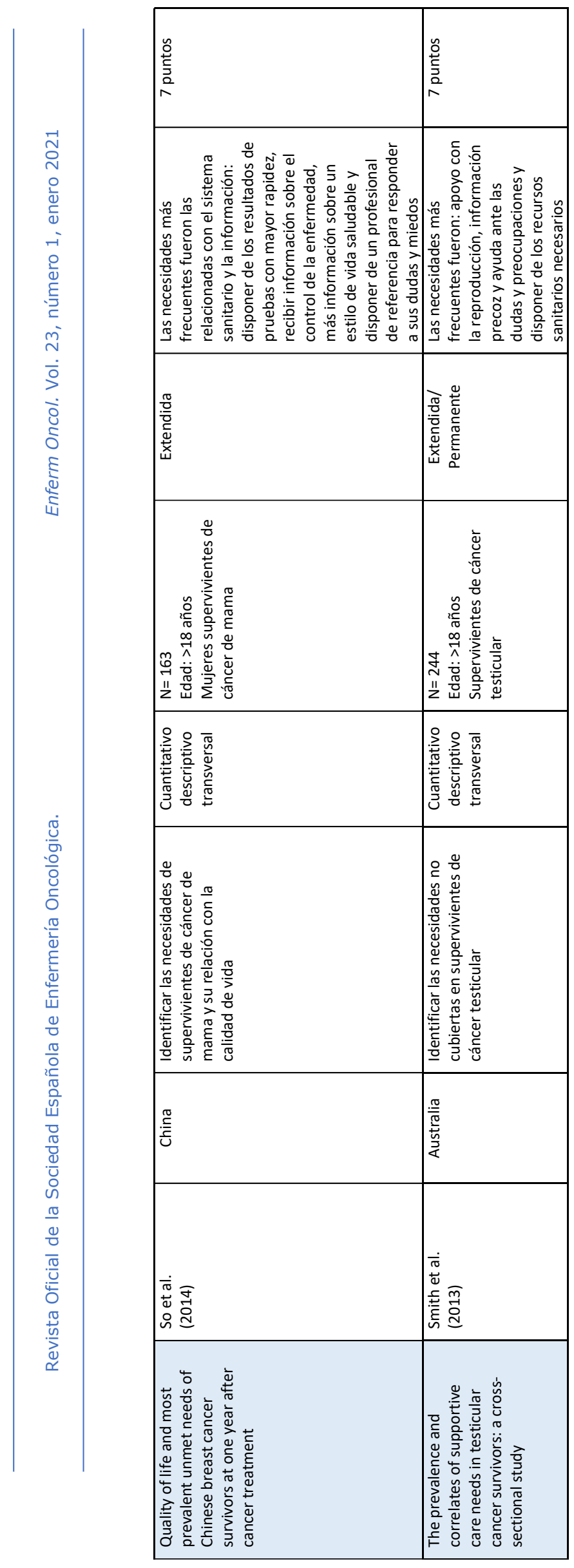

